TITLE:

\title{
Biaxial magnetic alignment in twinned $\mathrm{REBa}[2] \mathrm{Cu}[3] \mathrm{O}[y]$ superconductors
}

$\operatorname{AUTHOR}(\mathrm{S}):$

Horii, S; Nishioka, T; Arimoto, I; Fujioka, S; Doi, T

\section{CITATION:}

Horii, S ... [et al]. Biaxial magnetic alignment in twinned REBa[2]Cu[3]O[y]superconductors. Superconductor Science and Technology 2016, 29(12): 125007.

\section{ISSUE DATE:}

2016-10-13

URL:

http://hdl.handle.net/2433/217468

\section{RIGHT:}

This is an author-created, un-copyedited version of an article accepted for publication in 'Superconductor Science and Technology'. The publisher is not responsible for any errors or omissions in this version of the manuscript or any version derived from it. The Version of Record is available online at http://dx.doi.org/10.1088/0953-2048/29/12/125007.; The full-text file will be made open to the public on 13 October 2017 in accordance with publisher's 'Terms and Conditions for Self-Archiving'.; この論文は出版社版でありません。引用の際には出版社版をご確認ご利用ください。; This is not the published version. Please cite only the published version. 


\title{
Biaxial magnetic alignment in twinned $\mathrm{REBa}_{2} \mathrm{Cu}_{3} \mathrm{O}_{\mathrm{y}}$ superconductors
}

\author{
S Horii ${ }^{1}$, T Nishioka $^{1}$, I Arimoto ${ }^{1}$, S Fujioka ${ }^{1}$, and T Doi $^{1}$ \\ ${ }^{1}$ Graduate School of Energy Science, Kyoto University, Yoshida-Honmachi, Sakyo-ku, \\ Kyoto 606-8501, Japan \\ E-mail: horii.shigeru.7e@kyoto-u.ac.jp
}

\begin{abstract}
Biaxial magnetic alignment of $\mathrm{REBa}_{2} \mathrm{Cu}_{3} \mathrm{O}_{\mathrm{y}}(\mathrm{RE} 123, \mathrm{RE}=\mathrm{Y}, \mathrm{Nd}, \mathrm{Sm}$, Dy, and Er) superconductor powders containing twin microstructures was demonstrated. Appropriate choice of RE effectively improved the degrees of in-plane and $c$-axis orientation in RE123 powder samples aligned under modulated rotating magnetic fields at room temperature. From the relationship between the magnetic field strength and the degrees of orientation, it is concluded that heavy RE ions induced the improvement of the in-plane magnetic anisotropies in RE123 grains with twin microstructures.
\end{abstract}

\section{Introduction}

The rare-earth (RE)-based cuprate superconductor $\mathrm{REBa}_{2} \mathrm{Cu}_{3} \mathrm{O}_{y}$ (RE123) shows critical temperature $\left(T_{\mathrm{c}}\right)$ with approximately $90 \mathrm{~K}$, and its $T_{\mathrm{c}}$ is higher than the boiling temperature of liquid nitrogen $(77.3 \mathrm{~K})$. RE123 possesses a layered crystal structure stacked along the $c$-axis, which is including superconducting $\mathrm{CuO}_{2}$ planes spread along the $a b$-plane and a one-dimensional $\mathrm{CuO}$ chain running parallel to the $b$-axis. Due to low electromagnetic anisotropy of carriers induced by the metallic $\mathrm{CuO}$ chain as a blocking layer of RE123 with $y$ 7 , RE123 shows an intrinsically higher irreversibility field $\left(H_{\text {irr }}\right)$ at $77 \mathrm{~K}$ as compared with those for other cuprate superconductors [1]. One recognizes that RE123 compounds are practical superconductors not only for electric power cables but also for magnet application. However, due to the short coherence length and $d$-wave symmetry of the Cooper pair in cuprate superconductors, the intergrain critical current density (intergrain $J_{c}$ ) of the $c$-axis-oriented RE123 bicrystal decreases significantly with an increase in the misorientation angle at the grain boundary[2]. Therefore, a biaxial grain orientation is required in addition to the formation of a densified microstructure to achieve practical in-field and self-field $J_{\mathrm{c}}$ properties. Epitaxial growth methods, such as thin film growth on single-crystalline substrates [3] and melt-solidification using seed crystals [4], have been adopted to obtain biaxially oriented RE123 materials thus far.

Recently, a biaxial grain-orientation technique using a frequency-modulated rotating magnetic field, which is a technique for simultaneously aligning both the easy and hard axes of magnetization using a modulated rotation magnetic field (MRF), has been developed [5]. In principle, this technique is extensively applied for various polycrystalline functional materials that have triaxial magnetic anisotropy with sufficiently large differences in magnetic susceptibilities $(\chi)$ along crystallographic axes. Due to magnetic field energy proportional to the square of $H$, number of substances appropriate for the magnetic alignment are expected to increase remarkably by the use of 10-tesla-class high field magnets. At the current stage, cryogen-free superconducting magnets [6] which can generate $\sim 10 \mathrm{~T}$ have been available commercially. In practice, the biaxial orientation of a twin-free $\mathrm{Y}_{2} \mathrm{Ba}_{4} \mathrm{Cu}_{7} \mathrm{O}_{y}$ (Y247: $y \sim 15$ ) superconductor [7-9], which is an analog compound of RE123, with high degrees of in-plane and $c$-axis orientations below $2^{\circ}$, has been achieved in epoxy resin at room temperature under an MRF of $12 \mathrm{~T}[8,9]$ as a proof of principle. Our group has also reported the biaxial orientation in epoxy resin at room temperature for twin-free $\mathrm{REBa}_{2} \mathrm{Cu}_{4} \mathrm{O}_{8}$ (RE124) superconductors [10] and the triaxial magnetic anisotropies depending on the type of RE ions for RE124 [10] and 
$\mathrm{RE}_{2} \mathrm{Ba}_{4} \mathrm{Cu}_{7} \mathrm{O}_{\mathrm{y}}[11]$ at room temperature.

On the other hand, in the case of practical RE123 superconductors, one can predict that their biaxial grain alignment will be difficult to achieve. This is because the RE123 crystal or grain has twin microstructure [12] originating from the tetragonal-orthorhombic transition [13] corresponding to an increase of the nonstoichiometric oxygen content [14]. The RE123 grain containing the twin microstructure consists of two different types of domains in which the $c$-axes are parallel and the $a$-axes (or $b$-axes) are orthogonal, probably leading to the reduction or disappearance of magnetic anisotropy in the $a b$-plane direction at the grain level. However, the development of a magnetic biaxial alignment technique in RE123 is obviously important for demonstrating the further versatility of the novel magneto-scientific method. In the present study, we show the biaxial grain orientation of RE123 powders with twin microstructures by magnetic alignment as a proof of principle and clarify the dependences of the degrees of orientation on the magnetic field and the type of RE ions.

\section{Experimental details}

RE123 polycrystals were synthesized by standard solid-state reaction in air. The starting materials, $\mathrm{RE}_{2} \mathrm{O}_{3}(\mathrm{RE}=\mathrm{Y}, \mathrm{Nd}, \mathrm{Sm}$, Dy, and $\mathrm{Er}), \mathrm{BaCO}_{3}$, and $\mathrm{CuO}$, were weighed in a molar ratio of $1: 2: 3$ and ground in ethanol. The mixed powders were calcined at $860^{\circ} \mathrm{C}$ and $900^{\circ} \mathrm{C}$. Then, they were pelletized and sintered at $920^{\circ} \mathrm{C}$ for $24 \mathrm{~h}$. Y124 single crystals with $\sim 50$ $\mu \mathrm{m}$ in size were grown by a flux method in the air using $\mathrm{KOH}$ as a flux medium $[10,15,16]$. Details of the crystal growth have been reported elsewhere [10]. The obtained RE123 pellets and Y124 crystals were crushed in agate mortar to fabricate powders for magnetic alignment. A scanning electron microscope was used to determine the average diameters $(d)$ of the RE123 and Y124 powders, which were 1.5-3.0 $\mu \mathrm{m}$.

The obtained power was mixed with epoxy resin at a weight ratio of powder : resin = $0.2 \mathrm{~g}: 2.0 \mathrm{~g}$. The epoxy resin containing the RE123 or Y124 powder was cured for more than $12 \mathrm{~h}$ at room temperature in an MRF. In detail, the specimens were horizontally rotated at two different steps in a static field $\left(H_{\mathrm{a}}\right)$ of $\mu_{0} \mathrm{H}_{\mathrm{a}}=0,0.5,1,3,5$, and $10 \mathrm{~T}$ applied along the transverse direction. At angles of $0^{\circ}$ and $180^{\circ}$, the powder samples of RE123 and Y124 were rested for $2 \mathrm{~s}$, whereas the rotation process with $60 \mathrm{rpm}$ was applied at other angle regions. The sample's original angle, $0^{\circ}$, was defined with regard to a direction normal to the $\alpha$ plane (see Fig. 1 ) of the sample, which was parallel to the transverse $H_{a}$ direction. For cured resins containing magnetically aligned powders, XRD measurements were performed for the $\alpha, \beta$, and $\gamma$ planes (see Fig. 1). The easy, secondary easy, and hard axes oriented in an MRF could be determined from the XRD patterns of the $\alpha, \beta$, and $\gamma$ planes, respectively. To determine the degrees of the $c$-axis and in-plane orientations for the magnetically oriented powder samples, X-ray rocking curves (XRCs) and pole figures were examined. The full width at half maximum $(\Delta \omega)$ value for each XRC was obtained as the degree of orientation for a magnetically aligned powder sample.

\section{Results and discussion}

Figure 2(a) shows XRD patterns at the $\alpha$ plane for the RE123 powder samples aligned in the MRF with $\mu_{0} H_{\mathrm{a}}=10 \mathrm{~T}$, together with that at the $\alpha$ plane for the twin-free Y124 powder sample aligned in the MRF of $10 \mathrm{~T}$ for reference. In the case of Y124 and RE123 with RE=Y, $\mathrm{Nd}, \mathrm{Sm}$, and Dy, only (00l) peaks were clearly enhanced, which indicates the first easy axis of magnetization parallel to the $c$-axis. On the other hand, in the case of Er123, the ( $h 00)$ and $(0 \mathrm{k} 0)$ peaks were simultaneously enhanced. However, intensities of the $(0 k 0)$ peak were always larger than those of the (h00) peak; therefore, the first easy axis for Er123 was estimated to be the $b$-axis. These results were consistent with the results obtained from RE123 aligned under static magnetic fields [17-20]. 
Figure 2(b) shows XRD patterns at the $\gamma$ plane for the RE123 powder samples aligned in the MRF of $10 \mathrm{~T}$, together with that at the $\gamma$ plane for the twin-free Y124 powder sample aligned in the MRF of $10 \mathrm{~T}$ for reference. In the case of Er123, only (00l) peaks were clearly enhanced, which indicates the hard axis of magnetization parallel to the $c$-axis. On the other hand, in the case of Y124 and RE123 with RE=Y, Nd, Sm, and Dy, the (h00) and (0k0) peaks were simultaneously enhanced. However, intensities of the $(0 \mathrm{k} 0)$ peak were always larger than those of the ( $h 00)$ peak, leading to the hard axes parallel to the $b$-axis for RE123 with RE=Y, Nd, Sm, and Dy. From XRD patterns at the $\beta$ planes (not shown in this article) in addition to the results of Figs. 2(a) and 2(b), it was suggested that a biaxial orientation was basically achieved for all five powder samples of RE123 with twin microstructures. Here, it should be noted that in Fig. 2, the (110) peaks also appeared only for the RE123 powder samples in XRD patterns at the planes where both $(h 00)$ and $(0 k 0)$ peaks were enhanced, and intensities of the (110) peak depended on the type of RE in RE123. As shown in Fig. 2(b), only ( $0 k 0)$ peaks were strongly enhanced for the Y124 powder sample, and the co-existence of (h00) and (0k0) peaks and the appearance of the (110) peak were not observed. As discussed later, these phenomena observed only in RE123 were due to twin microstructures formed in RE123 powders.

To understand the details of biaxial magnetic alignment for the RE123 and Y124 powder samples aligned in the MRF of $10 \mathrm{~T}$, pole figure measurements using (103) for RE123 and (017) for Y124 were performed at the planes where the $(00 l)$ peaks were enhanced. Figures 3(a)-3(f) show the (103) pole figures for the RE123 (RE=Y, Nd, Sm, Dy, and Er) powder samples aligned in the MRF of $10 \mathrm{~T}$ and the (017) pole figure for the Y124 powder sample aligned in the MRF of $10 \mathrm{~T}$. For comparison, the (103) pole figure at the $\alpha$ plane for the Dy123 powder sample prepared without applying the magnetic field was also shown in Fig. 3(g). As shown in Fig. 3(f), in the case of twin-free Y124, sharp two-fold symmetric peaks were observed. This reflects the biaxial orientation of orthorhombic Y124 grains without twin microstructures. However, in the case of RE123 in Figs. 3(a)-3(e), four-fold symmetric peaks and a broadening of the peaks to the rotation angle appeared. Clearly, the co-existence of $(h 00)$ and $(0 k 0)$ peaks in Fig. 2 and the four-fold symmetric peaks in Fig. 3 were correlated with the generation of twin microstructures in RE123. When one focuses on the pole figure in Fig. 3(g), distribution of the diffraction peaks showed plateau-shape. This means random orientation of Dy123 grains was achieved in the case of $\mu_{0} H_{\mathrm{a}}=0 \mathrm{~T}$. Furthermore, similar results were obtained for other RE123 (RE = Y, Nd, Sm, and Er) powder samples prepared without applying the magnetic field. It was clearly found that the sample rotation under $\mu_{0} H_{\mathrm{a}}=0 \mathrm{~T}$ was not effective for improving the grain orientation. Therefore, the pole figures in Figs. 3(a)-3(e) strongly indicate that biaxial grain orientation was achieved by applying the MRF of $10 \mathrm{~T}$ even for RE123 powders with twin microstructures, and the results in Fig. 2 and Fig. 3 can be understood in terms of the biaxial orientation of RE123 grains containing two domains in which the $c$-axes are parallel and the $a$-axes (or $b$-axes) are orthogonal.

Another difference between RE123 and Y124 in Fig. 3 is the broadening of the peaks in the direction of the rotation angle. Especially for Y123, Sm123, and Er123, ring-like diffraction emerged in addition to the four peaks. This result indicates that two different components of magnetic alignment were contained in the powder samples of Y123 and Sm123: biaxial-oriented grain and $c$-axis- (uniaxial or weakly biaxial) oriented grain. One can recognize that the existence of the $c$-axis-oriented grains leads to the appearance of the (110) diffraction peak at planes where both $(h 00)$ and $(0 k 0)$ peaks were enhanced. From the results in Fig. 2 and Fig. 3, the first easy, second easy, and hard axes of magnetization for RE123 at room temperature in the molecule level can be determined. The magnetization axes for RE123 were summarized in Table 1 , together with ionic radii of $\mathrm{RE}^{3+}$ with $\mathrm{CN}=8$ (CN; coordination number)[23] for reference. For RE123 with RE=Y, Nd, Sm, and Dy, the first easy, second easy, and hard axes of magnetization were $c$-, $a$-, and $b$-axes, respectively. However, for Er123, the first easy, second easy, and hard axes of magnetization were $b-, a-$, and $c$-axes, respectively. 
These magnetization axes in RE123 corresponded with those of twin-free RE124 (RE=Y, Nd, Sm, Dy, and Er)[10,21] and twin-free $\mathrm{RE}_{2} \mathrm{Ba}_{4} \mathrm{Cu}_{7} \mathrm{O}_{y}$ (RE247: $\mathrm{RE}=\mathrm{Y}$, Dy, and Er) [11] for same $\mathrm{RE}$. That is, the single-ion magnetic anisotropies[24] of $\mathrm{RE}^{3+}$ are dominant for $\mathrm{RE}-\mathrm{Ba}-\mathrm{Cu}-\mathrm{O}$ with heavy $\mathrm{RE}$ and contribution of magnetic anisotropies of $\mathrm{Cu}$ ions are large for $\mathrm{RE}-\mathrm{Ba}-\mathrm{Cu}-\mathrm{O}$ with light RE.

To clarify the changes in the degrees of grain orientation depending on $H_{\mathrm{a}}$ and the type of RE, XRCs were examined for the RE123 and Y124 powder samples aligned in the MRFs of $\mu_{0} H_{\mathrm{a}}=0.5,1,3,5$, and $10 \mathrm{~T}$ using the diffraction peaks of ( $\left.00 l\right)$ ( $l=5$ for RE123, $l=12$ for Y124) and (020). The degrees of the $c$-axis and in-plane orientations for the powder samples were determined as $\Delta \omega$ values for the XRCs at the $(00 l)$ and $(020)$ peaks, $\Delta \omega^{\text {-axis }}$ and $\Delta \omega^{\text {n-plane }}$, respectively, for the tilt of the samples to the $a$-axis direction (or the $\beta$ plane). Note that, for $\mathrm{RE}=\mathrm{Y}$, Dy, and Er, two $\Delta \omega-H_{\mathrm{a}}$ curves were obtained individually using two different sintered pellets of RE123. Figure 4(a) shows the $H_{\mathrm{a}}$ dependence of $\Delta \omega^{\text {-axis }}$ for the magnetically aligned powder samples of RE123 (RE=Y, Nd, Sm, Dy, and Er) and Y124 to evaluate changes in the degrees of the $c$-axis orientation. In the case of $\mu_{0} H_{\mathrm{a}} \geq 1 \mathrm{~T}$, high degrees of the $c$-axis orientation with $\Delta \omega^{\text {-axis }}<4^{\circ}$ were accomplished for all of the oriented RE123 and Y124 powder samples. This indicates that $c$-axis magnetic anisotropy $\left(\chi_{c}-\chi_{a b}\right)$ sufficient for the $c$-axis grain orientation under the MRF of $1 \mathrm{~T}$ is preserved and is not affected by the twin microstructure. On the other hand, in the cases of $\mu_{0} H_{\mathrm{a}}=0.5 \mathrm{~T}$, the degrees of $c$-axis orientation were clearly lowered for Y123, and $\Delta \omega^{\text {-axis }}<4^{\circ}$ was maintained for RE123 (RE= Nd, Sm, Dy, and Er).

Figure 4(b) shows the $H_{\mathrm{a}}$ dependence of $\Delta \omega^{\text {n-plane }}$ for the magnetically aligned powder samples of RE123 (RE=Y, Nd, Sm, Dy, and Er) and Y124 to evaluate changes in the degrees of the in-plane orientation. A striking feature is that the $\Delta \omega^{\text {in-plane }}$ values depended largely on the type of RE and the phase, even for powder samples aligned under the MRF of $10 \mathrm{~T}$. In detail, the lowest $\Delta \omega^{\text {in-plane }}$ value with $\Delta \omega^{\text {in-plane }}<3^{\circ}$ was achieved for Y124 without a twin microstructure, whereas the $\Delta \omega^{\text {n-plane }}$ for RE123 with a twin microstructure showed a relatively wider range of $\Delta \omega^{\text {n-plane }}=3-8^{\circ}$. Our group [10] also reported $\Delta \omega^{\text {j-plane }}=2.0,5.0,2.6$, and $3.2^{\circ}$ for the RE124 (RE=Y, Sm, Dy, and Er) powder samples aligned under the MRF of $10 \mathrm{~T}$, respectively. That is, in the magnetic alignment in the MRF of $10 \mathrm{~T}$, the in-plane orientation showed obviously higher degrees of the inplane orientation in RE124 without twin microstructures than in RE123 with twin microstructures. However, $\Delta \omega^{\text {jn-plane }} \sim 3^{\circ}$ and $\Delta \omega^{\text {-axis }}<$ $1^{\circ}$ were achieved for Dy123 and Nd123, strongly indicating the high potential of the magnetic alignment using MRF as a biaxial alignment technique. Another interesting feature is the difference in the $H_{\mathrm{a}}$ dependence of $\Delta \omega^{\mathrm{j} \text {-plane }}$. In the cases of Dy123 and Er123, their respective $\Delta \omega^{\text {j-plane }}$ values were almost independent of $H_{\mathrm{a}}$, and those for $\mu_{0} H_{\mathrm{a}} \geq 1 \mathrm{~T}$ showed similar values. In particular, $\Delta \omega^{\mathrm{n} \text {-plane }}<4^{\circ}$ and $\Delta \omega^{\text {-axis }} \sim 1^{\circ}$ were achieved even for $\mu_{0} H_{\mathrm{a}}=1 \mathrm{~T}$ for Dy123, strongly suggesting that biaxial grain alignment with high orientation degrees is achieved even by the usage of permanent magnets and non-superconducting electromagnets. On the other hand, the $\Delta \omega^{\text {in-plane }}$ values decreased monotonically with the decrease in $H_{\mathrm{a}}$ for Y123, Sm123, and Y124, and decreased below $5 \mathrm{~T}$ for $\mathrm{Nd} 123$. The $\mathrm{Y}^{3+}$ ion does not contain $4 \mathrm{f}$ electrons, and light $\mathrm{RE}^{3+}$ ions show intrinsically lower single-ion magnetic anisotropies [10,19,20,24]. The decrease in $\Delta \omega^{\text {n-plane }}$ at higher $H_{\mathrm{a}}$ for RE123 with $\mathrm{RE}=\mathrm{Y}, \mathrm{Nd}$, and $\mathrm{Sm}$ can be explained in terms of the low magnetic anisotropies of RE ions in addition to the reduction of in-plane magnetic anisotropies at the grain level by the twin microstructures.

\section{Summary}

In conclusion, we attempted biaxial magnetic alignment in MRFs for RE123 powders. For the MRF of $10 \mathrm{~T}$, the biaxial alignment with $\Delta \omega^{\text {in-plane }}=3-8^{\circ}$ and $\Delta \omega^{c \text {-axis }} \sim 1^{\circ}$ was achieved despite the existence of twin microstructures in the grains of RE123. The $\Delta \omega^{\text {-axis }}$ values were independent of RE and $H_{\mathrm{a}}$ for $\mu_{0} H_{\mathrm{a}}>0.5 \mathrm{~T}$, whereas $\Delta \omega^{\mathrm{n} \text {-plane }}$ is sensitive to $H_{\mathrm{a}}$ and the type of 
RE. Consequently, the degrees of in-plane orientation were drastically improved by the appropriate choice of RE and the increase in $H_{\mathrm{a}}$. When one chooses Dy123 and Er123, higher degrees of the in-plane orientation with $\Delta \omega^{\text {n-plane }} \sim 4^{\circ}$ and $\Delta \omega^{\text {n-plane }}=7-8^{\circ}$ were achieved at the MRF of $1 \mathrm{~T}$, respectively. The present study suggests that the biaxial magnetic alignment technique enables the fabrication of biaxially oriented bulks and thick films of twinned RE123 on the basis of combination of the magnetic alignment and colloidal techniques [25-29]. In practice, our group attempted to fabricate biaxially oriented green compacts of twin-free Er124 by slip-casting under MRFs and clarified relationship between $\Delta \omega^{\text {n-plane }}$ and viscosity of slurry $(\eta)$ [30]. The increase in $\eta$ was found to be effective for improving $\Delta \omega^{\text {in-plane}}$, whereas the increase in $\mu_{0} H_{\mathrm{a}}$ for the slurry with lower $\eta$ seriously led to the increase in magnetic force, which was induced by $\chi$, magnetic field and magnetic gradient, for fine Er124 crystals with large value of $\chi\left(\sim 5 \times 10^{-3}\right.$ [31] in the case of Er247[32]) as a paramagnetic substance. Taking the formation of the twin microstructure in RE123 into account, the previous report suggests that appropriate choice of RE, synthesis of particle with single grain and control of grain size are more important to obtain highly biaxial oriented green compacts of RE123.

\section{Acknowledgments}

This work was partly supported by the Murata Science Foundation. 


\section{REFERENCES}

[1] Shimoyama J, Kitazawa K, Shimizu K, Ueda S, Horii S, Chikumoto N, and Kishio K 2003 J. Low Temp. Phys. 1311043

[2] Dimos D, Chaudhari P, Mannhart J and LeGoues F K 1988 Phys. Rev. Lett. 61219.

[3] Laibowitz R B, Koch R H, Chaudhari P and Gambino R J 1987 Phys. Rev. B 358821

[4] Chen Y L, Chan H M, Harmer M P, Todt V R, Sengupta S and Shi D 1994 Physica C 234 232

[5] Kimura T, Kimura F and Yoshino M 2006 Langmuir 223464

[6] Watanabe K, Awaji S, Fukase T, Yamada Y, Sakuraba J, Hata F, Chong C K, Hasebe T, and Ishihara M 1994 Cryogenics 34639

[7] Stains M, Genoud J, Mawdsley A, and Manojlovic V 1999 IEEE Trans. Appl. Supercond. 9 2284

[8] Fukushima T, Horii S, Ogino H, Uchikoshi T, Suzuki T S, Sakka Y, Shimoyama J and Kishio K 2008 Appl. Phys. Express 1111701

[9] Horii S, Ishihara A, Fukushima T, Uchikoshi T, Ogino H, Suzuki T S, Sakka Y, Shimoyama J and Kishio K 2009 Sci. Technol. Adv. Mater. 10014604

[10] Yamaki M, Horii S, Haruta M, and Shimoyama J 2012 Jpn. J. Appl. Phys. 51010107

[11] Fukushima T, Horii S, Uchikoshi T, Ogino H, Ishihara A, Suzuki T S, Sakka Y, Shimoyama J and Kishio K 2009 IEEE Trans. Appl. Supercond. 192961

[12] Hiroi Z, Takano M, Takeda Y, Kanno R and Bando Y 1988 Jpn. J. Appl. Phys. 27 L580

[13] Jorgensen J D, Veal B W, Kwok W K, Crabtree G W, Umezawa A, Nowicki L J, and Paulikas A P 1990 Phys. Rev. B 365731

[14] Kishio K, Shimoyama J, Hasegawa T, Kitazawa K and Fueki K 1987 Jpn. J. Appl. Phys. 26 L1228

[15] Song Y T, Peng J B, Wang X, Sun G L, and Lin C T 2007 J. Cryst. Growth 300263

[16] Sun G L, Song Y T, and Lin C T 2008 Supercond. Sci. Technol. 21125001

[17] Rango P De, Lees M, Lejay P, Sulpice A, Tournier R, Ingold M, Germi P, and Pernet M 1991 Nature 349770

[18] Farrel D E, Chandrasekhar B S, Deguire M R, Fang M M, Kogen V G, Clem J R, and Finnemore D K 1987 Phys. Rev. B 364025

[19] Ferreira J M, Maple M B, Zhou H, Hake R R, Lee B W, Seaman C L, Kuric M V, and Guertin R P 1988 Appl. Phys. A47, 105

[20] Ishihara A, Horii S, Uchikoshi T, Suzuki T S, Sakka Y, Ogino H, Shimoyama J, and Kishio K 2008 Appl. Phys. Express 1031701

[21] Horii S, et al., unpublished.

[22] Tamegai T, Watanage A, Oguro I, and Iye Y, 1987 Jpn. J. Appl. Phys. 26 L1304

[23] Shannon R D 1976 Acta Cryst. A32, 751

[24] Livingston J D, Hart H R Jr, and Wolf W P, 1988 J. Appl. Phys. 645806

[25] Horii S, Matsubara I, Sano M, Fujie K, Suzuki M, Funahashi R, Shikano M, Shin W, Murayama N, Shimoyama J, and Kishio K, 2003 Jpn. J. Appl. Phys. 427018

[26] Zhou Y, Matsubara I, Horii S, Funahashi R, Shikano M, Shimoyama J, Kishio K, Shin W, Izu N, and Murayama N, 2003 J. Appl. Phys. 932653

[27] Okamoto T, Horii S, Uchikoshi T, Suzuki T S, Sakka Y, Funahashi R, Ando N, Shimoyama J, and Kishio K, 2006 Appl. Phys. Lett. 89081912

[28] Suzuki T S, Uchikoshi T, and Sakka Y, 2006 Sci. Tech. Adv. Mater. 9356

[29] Tanaka S, Makiya A, Kato Z, Uchida N, Kimura T, and Uematsu K, 2006 J. Mater. Res. 21 703

[30] Yamaki M, Furuta M, Doi T, Shimyama J, and Horii S, 2014 Phys. Proc. 5862

[31] Magnetic susceptibility (volume) in SI unit is dimensionless.

[32] Horii S, Okuhira S, Yamaki M, Kishio K, Shimoyama J, and Doi T, 2014 J. Appl. Phys. 115 113908 
Table 1. The first easy, second easy, and hard axes of magnetization at room temperature for RE123. Ionic radii of $\mathrm{RE}^{3+}$ for $\mathrm{CN}=8[23]$ are shown for reference.

\begin{tabular}{cccccc}
\hline \hline & \multicolumn{5}{c}{ RE123 (ionic radii of $\mathrm{RE}^{3+}$ with $\left.\mathrm{CN}=8\right)$} \\
\cline { 2 - 6 } & $\mathrm{Y} 123$ & $\mathrm{Nd} 123$ & $\mathrm{Sm} 123$ & $\mathrm{Dy} 123$ & Er123 \\
$(0.116 \mathrm{~nm})$ & $(0.125 \mathrm{~nm})$ & $(0.122 \mathrm{~nm})$ & $(0.117 \mathrm{~nm})$ & $(0.114 \mathrm{~nm})$ \\
\hline 1st easy & $c$ & $c$ & $c$ & $c$ & $b$ \\
2nd easy & $a$ & $a$ & $a$ & $a$ & $a$ \\
hard & $b$ & $b$ & $b$ & $b$ & $c$ \\
\hline \hline
\end{tabular}




\section{Figure Captions}

\section{Figure 1}

Schematic of the relationship among $H_{\mathrm{a}}$, measured surfaces of $\mathrm{XRD}(\alpha, \beta, \gamma)$, and the rotation of a sample.

Figure 2

XRD patterns at the (a) $\alpha$ and (b) $\gamma$ planes for the RE123 (RE=Y, Nd, Sm, Dy, and Er) and Y124 powder samples aligned in the MRF of $10 \mathrm{~T}$.

Figure 3

(103) pole figures for the (a) Y123, (b) Nd123, (c) Sm123, (d) Dy123, and (e) Er123 powder samples aligned under the MRF of $10 \mathrm{~T}$. (f) (107) pole figure for the Y124 powder sample aligned in the MRF of $10 \mathrm{~T}$. (g) (103) pole figure for the Dy123 powder sample prepared without applying the magnetic field.

Figure 4

$H_{\text {a }}$ dependence of (a) $\Delta \omega^{\text {-axis }}$ and (b) $\Delta \omega^{\text {in-plane }}$ for the RE123 (RE=Y, Nd, Sm, Dy, and Er) and Y124 powder samples. Two curves for RE=Y, Dy, and Er were obtained individually from two different sintered pellets of RE123. 


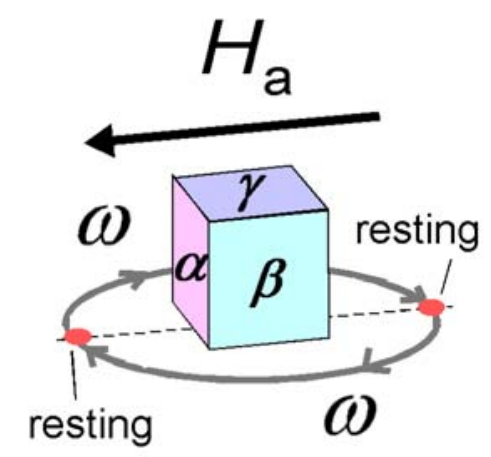

Figure 1 Horii et al. 
(a)

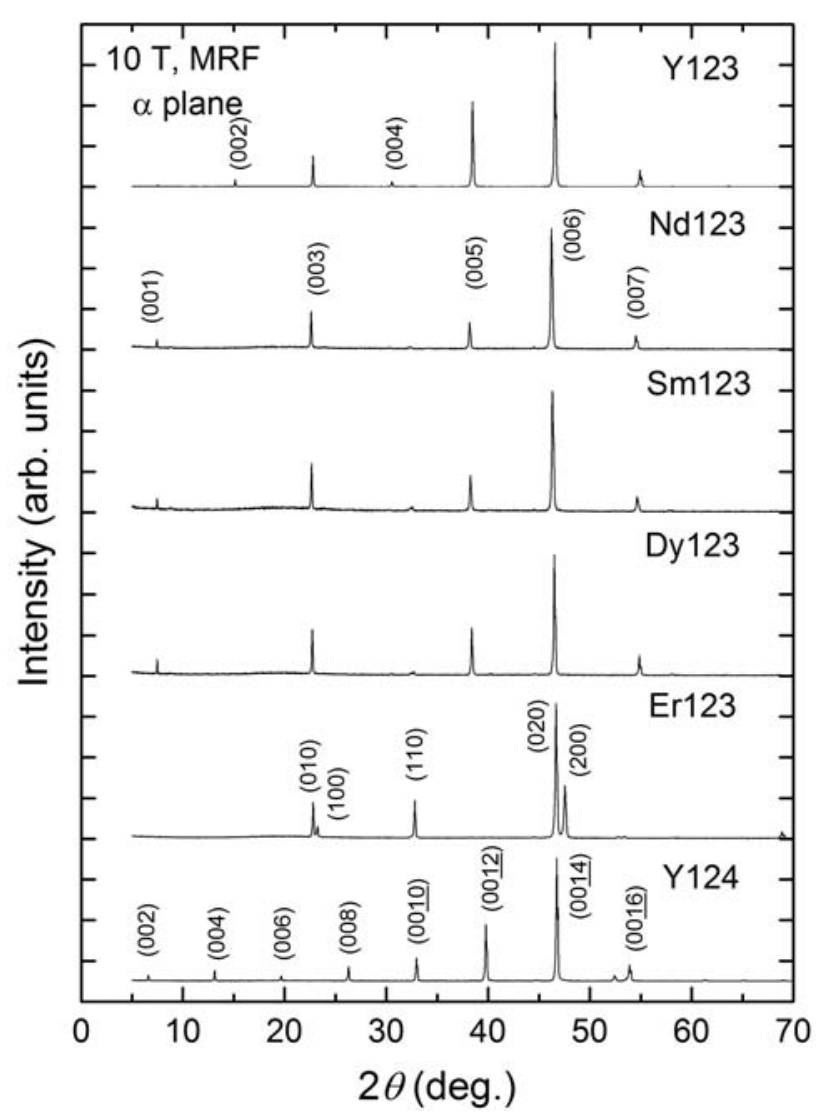

(b)

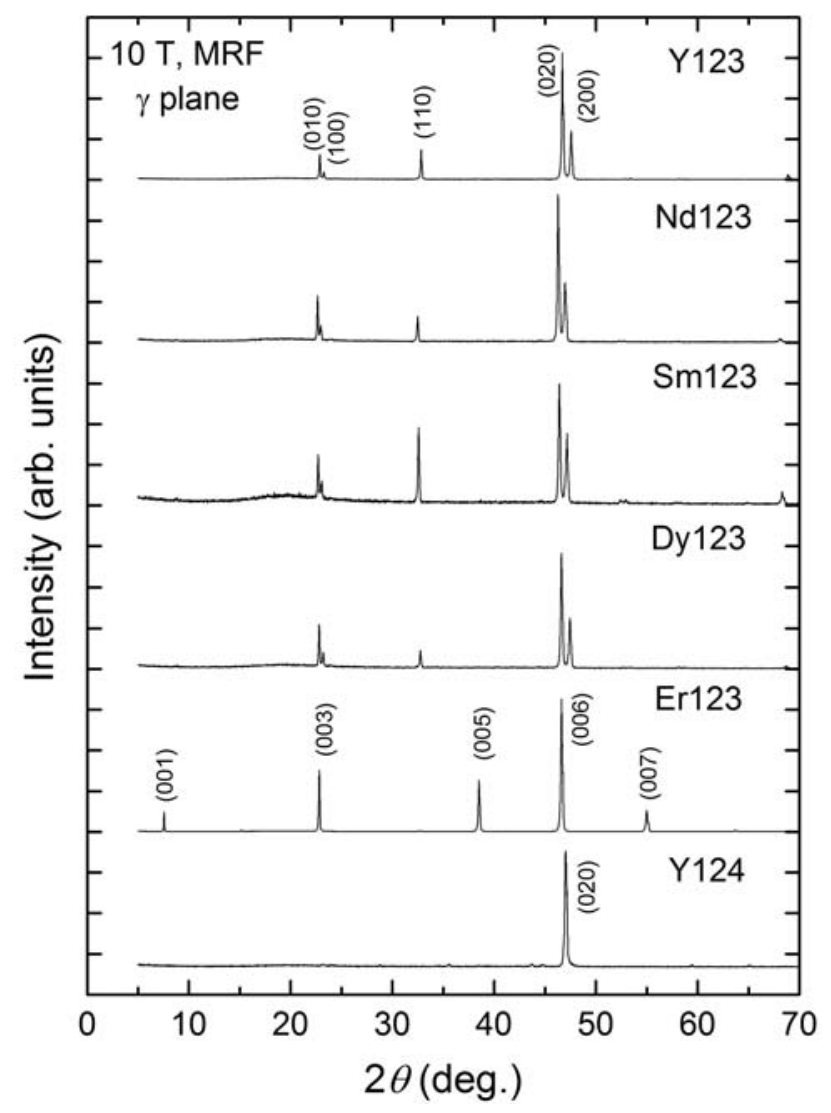

Figure 2 Horii et al. 
(a)

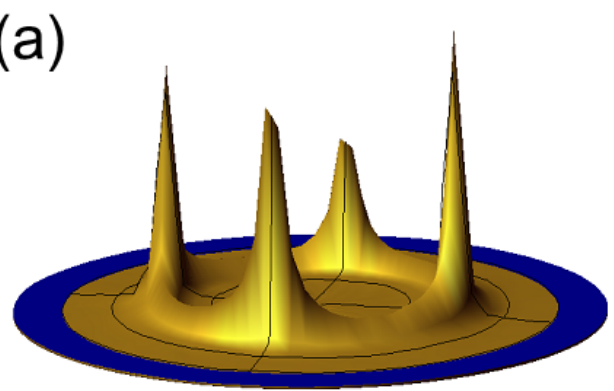

(c)

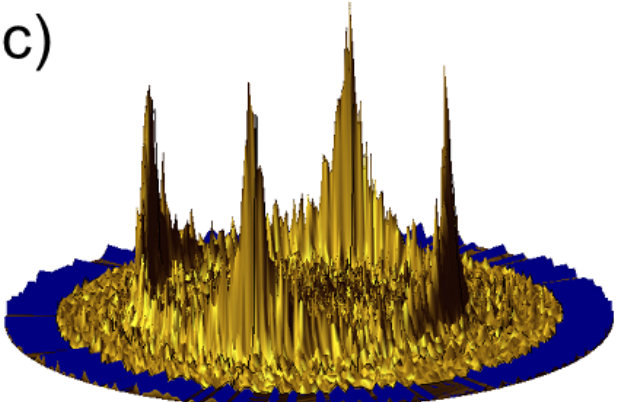

(e)

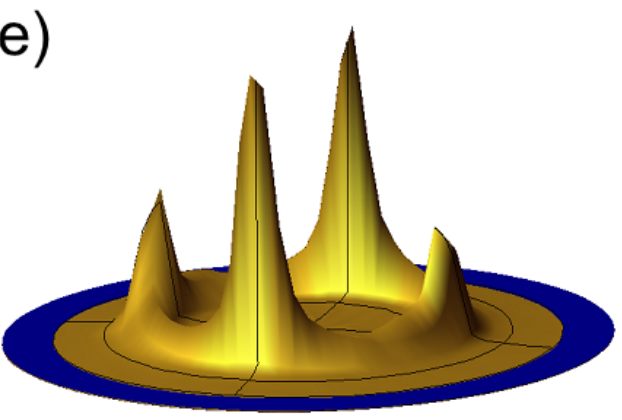

(g)

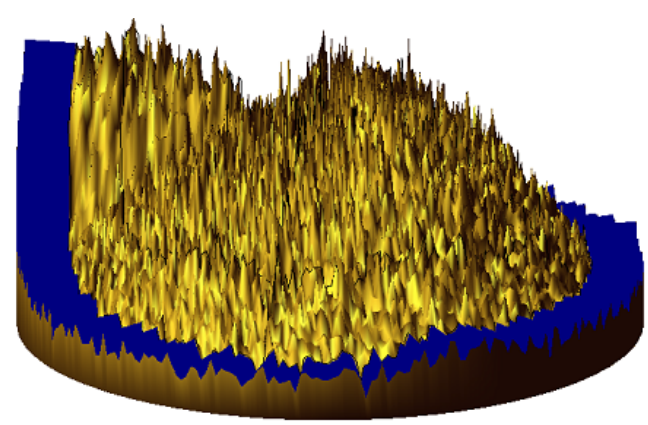

(b)

(d)

(f)

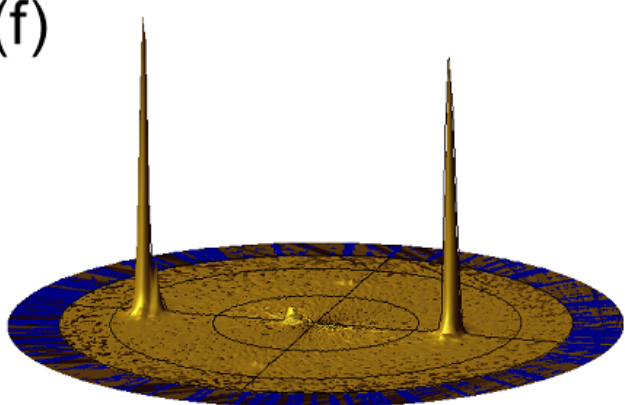

Figure 3 Horii et al. 
(a)

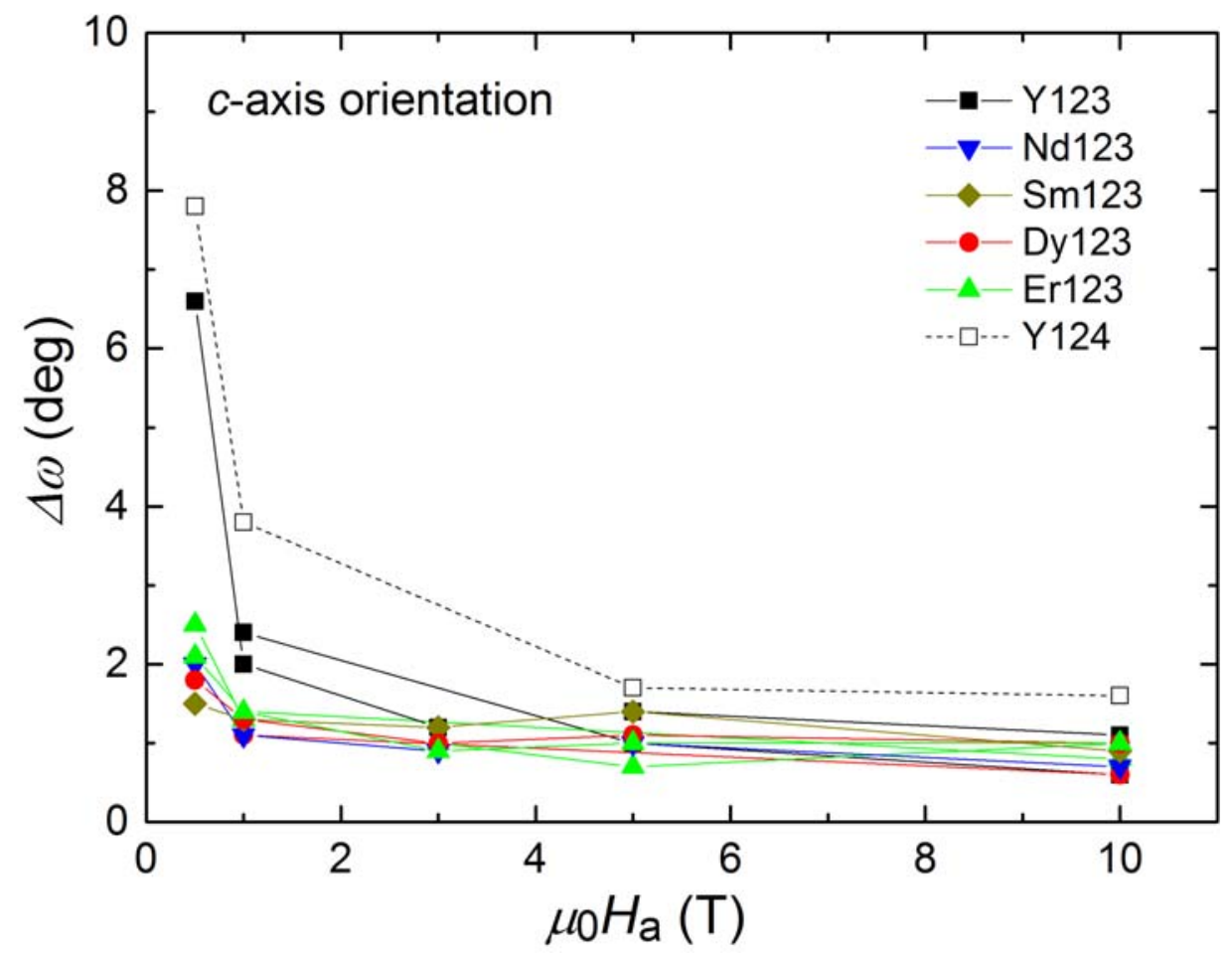

(b)

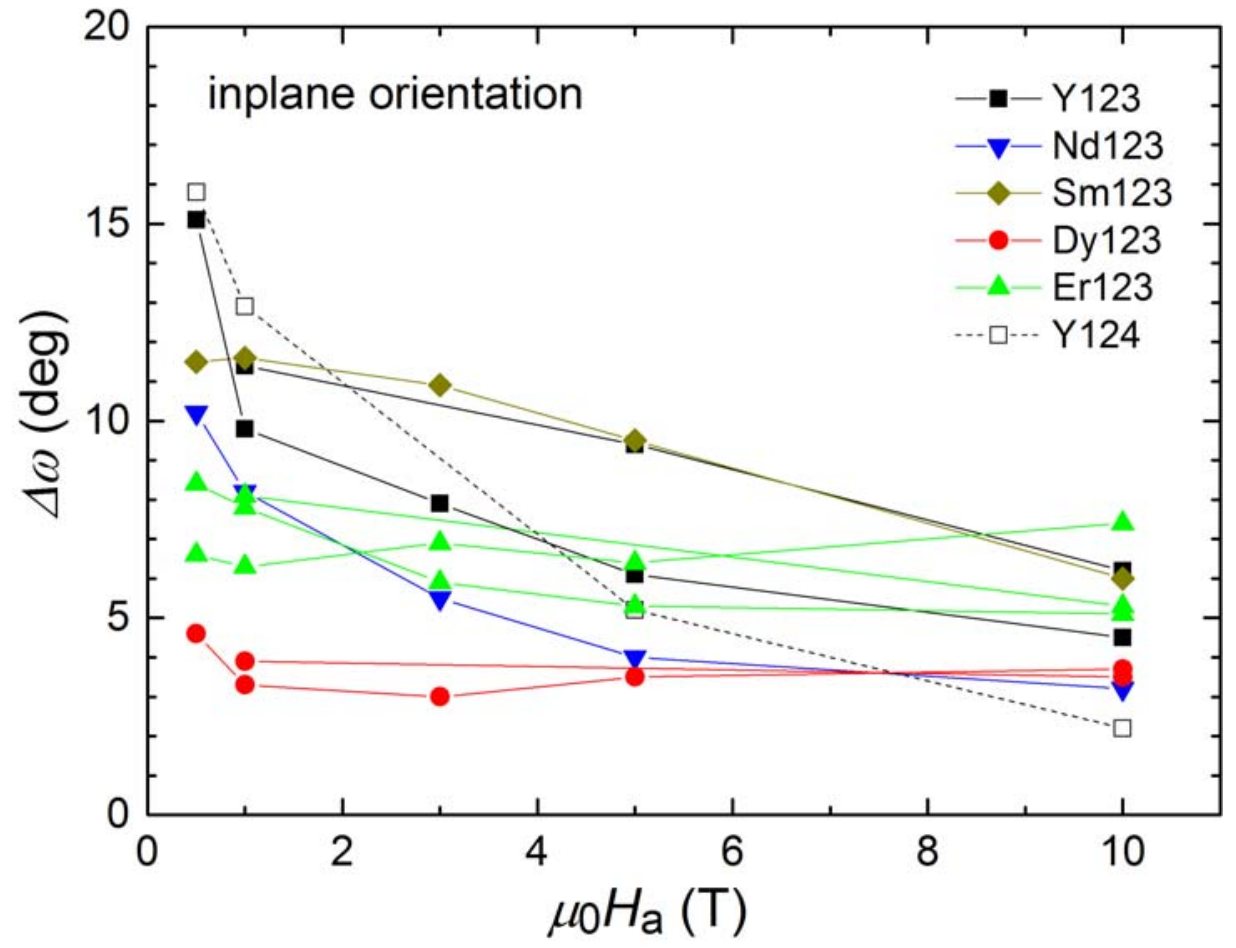

Figure 4 Horii et al. 